\title{
Nanofotónica en el análisis de biocombustibles
}

\author{
Nanophotonics to analyze biofuels \\ Beynor Antonio Paez-Sierra ${ }^{1 *} \quad$ Diana Maritza Marulanda Cardona ${ }^{2}$ \\ Recibido 26 de abril de 2016, aceptado 22 de abril de 2017 \\ Received: April 26, $2016 \quad$ Accepted: April 22, 2017
}

\begin{abstract}
RESUMEN
Actualmente uno de los retos energéticos es la búsqueda de alternativas para suplir anticipadamente el futuro agotamiento del petróleo a nivel mundial, y que además sean favorables con el medio ambiente. En especial los biocombustibles se han convertido en una de las propuestas más favorables para cubrir las actuales demandas del petróleo. Los biocombustibles son producidos de organismos vivos o de subproductos metabólicos (orgánicos o de desechos alimentarios). En este artículo se presenta una aplicación particular de la nanofotónica en el marco de la interacción de la luz con la materia denominada espectroscopia Raman de señal magnificada. Como muestra de control se considera el biodiésel y su mezcla con diésel, la razón se debe a la necesidad de establecer métodos de análisis durante el proceso de producción que podrían servir como cuantificador del grado de integridad al ser mezclado con diesel.
\end{abstract}

Palabras clave: Biocombustibles, biodiésel, SERS, espectroscopia Raman, nanofotónica, sensórica.

\begin{abstract}
Currently one of the energetic challenges is to look for environmentally friendly alternatives that would fulfill in advance the future shortage of oil in the world. Especially, biofuels have turned into one of the most favorable proposals to overcome current demands of the oil. Biofuels are produced from living organisms or from metabolic by-products (organic or food waste products). In this paper a particular application of nanophotonics in the frame of the interaction between light and matter through SurfaceEnhanced Raman spectroscopy (SERS) is addressed. As a test prove a Biodiésel sample is considered, the reason relies on the necessity to establish analysis procedures either to be performed during the production or as a quantifying tool to probe integrity during the blending process with diesel.
\end{abstract}

Keywords: Biofuels, biodiésel, nanophotonics, Raman spectroscopy, sensorics.

\section{INTRODUCCIÓN}

Las ventajas ambientales y económicas del biodiésel o B100 como combustible de motores han sido ampliamente discutidas en la literatura [1-3]. El Biodiésel tiene la ventaja de ser más favorable con el medio ambiente en comparación con los combustibles minerales, además presenta baja toxicidad, suministro sostenible, productos de degradación inofensivas y ser un subproducto importante de ciertas cosechas agrícolas. Aunque en medio de las ventajas mencionadas, también

1 Departamento de Física, Grupo NanoFab. Universidad Militar Nueva Granada. Cajicá, Sede Campus Nueva Granada. kilómetro 2 vía Cajicá. Zipaquirá, Colombia. E-mail: beynor.paez@unimilitar.edu.co

2 Facultad de Ingeniería. Universidad Militar Nueva Granada. Carrera 11101 80. Bogotá, Colombia.

E-mail: diana.marulanda@unimilitar.edu.co

* Autor correspondencia 
se presentan puntos débiles como su degradación debido a baja estabilidad química, influenciada principalmente por procesos de oxidación.

En la actualidad Colombia ha adoptado una política para incentivar la producción biocombustibles i.e. etanol y el biodiésel, como anticipación a la evidente disminución de reservas de petróleo. En especial el biodiésel es una excelente alternativa para prevenir situaciones de crísis relacionadas con el petróleo puesto que la relación de producción/consumo es favorable. La materia prima del biosiésel son los aceites vegetales o grasas animales, el cual resulta de la transesterificación de los trigliceridos al ser contenidos por un alcohol de cadena corta como el metanol [2-4].

A nivel nacional la distribución de diésel proveniente del petróleo, se mezcla con biodiésel. El resultado de la mezcla se ha denotado por los códigos B0, B2, B8 y B10 siendo "B" biodiésel seguido por el número que indica su participación porcentual en la mezcla con diésel. En la Figura 1 se presenta la distribución a nivel nacional de la participación porcentual de biodiésel en diésel. De la Figura 1 se observa que aproximadamente la mitad del territorio nacional presenta una participación del $10 \%$ de

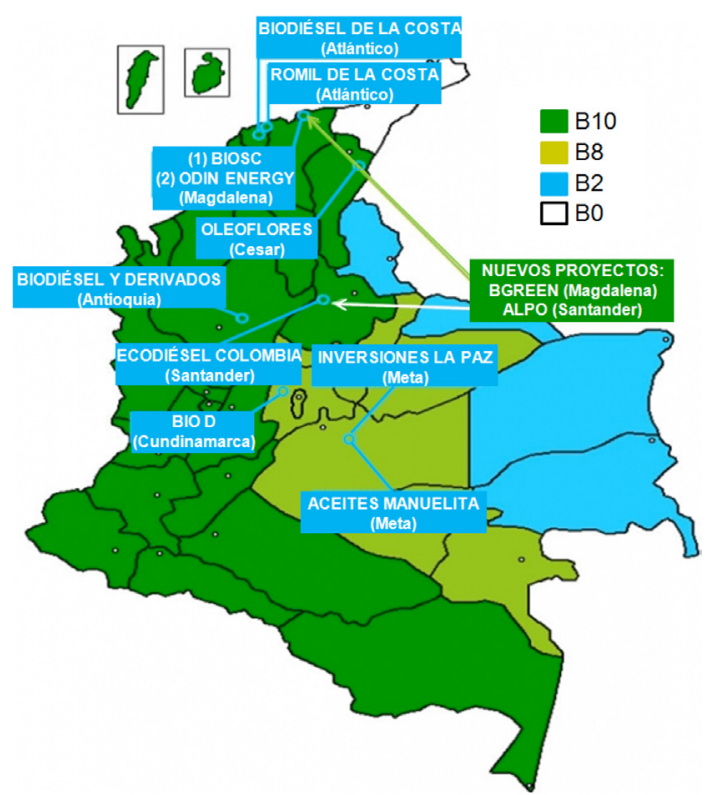

Figura 1. Distribución correspondiente al 2016 de la mezcla de biodiésel en diésel en Colombia [5]. biodiésel en diésel, mientras que para el resto del país la presencia de biodiésel en la mezcla es del $8 \%, 2 \%$ y $0 \%$.

Por consiguiente el mercado de los biocombustibles está en constante aumento lo que hace que también se plantee la posibilidad de aumentar el porcentaje de biodiésel en diésel. Sin embargo, actualmente no se cuenta con un plan de muestreo de la calidad del diésel que se expende en el país y que permita conocer exactamente los porcentajes de biodiésel a los cuales llega el combustible al consumidor. También es importante tener un control del efecto del biocombustible sobre la integridad física y desempeño del motor [6], ya que luego del proceso de combustión quedan trazas de nanopartículas que podrían disminuir su vida útil. Por esta razón es inminente la necesidad de contar con una herramienta de fácil uso y que permita identificar de manera inmediata la cantidad porcentual de biodiésel en diésel. Es en este aspecto donde el diagnóstico no destructivo de los biocombustibles sería de gran apoyo para el productor y el consumidor. Con este artículo queda en evidencia que una herramienta nanofotónica puede ser una solución complementaria al reto global para la determinación del contenido porcentual de biodiésel en diésel. Es aquí donde las técnicas basadas en la interacción de la luz con la materia son de gran utilidad [7-11].

El término nanofotónica hace referencia a la manipulación o utilización de la luz visible o invisible en la escala de los nanómetros $(1 \mathrm{~nm}$ $=10^{-9} \mathrm{~m}$, esto es 100.0000 más pequeño que el espesor de un cabello humano). Por ejemplo, la luz que emite una pantalla tipo OLED (Organic Light emitting Diode) se hace desde estructuras que no superan los $100 \mathrm{~nm}$ de espesor [12]. La luz blanca proveniente del sol al llegar a la atmósfera interactúa con diversas moléculas, cuyo tamaño es menor que $10 \mathrm{~nm}$, provocando un fenómeno de dispersión, esto hace que veamos el firmamento de color azul. La luz tiene la propiedad de interactuar molecularmente con la materia en todos sus estados i.e. sólido, líquido y gaseoso. Por tanto, la investigación y desarrollo de los fenómenos que implican el comportamiento de la luz debido a su interacción con la materia a nivel atómico o molecular se llama nanofotónica [13]. Las moléculas poseen una huella molecular o espectro que las distingue unas de otras, es aquí donde la nanofotónica se 
convierte en un instrumento indispensable para la distinción de sustancias. A manera de ilustración imagine dos frascos transparentes y herméticamente sellados y suponga que quiere saber su contenido sin destaparlos. En primera aproximación sería difícil distinguirlos visualmente porque ambos se ven transparentes. Por tanto, una aplicación basada en nanofotónica sería de gran ayuda, puesto que la luz dispersada portaría la información de las moléculas de cada uno de los líquidos.

Las técnicas ópticas suministran ventajas únicas y complementarias al no ser invasivas. Además, requieren de bajas energías de excitación, y posibilidad de analizar la estructura molecular bajo condiciones no ionizantes [14].

Entre las diversas técnicas de caracterización óptica la espectroscopia Raman desempeña una función importante para determinar las propiedades vibracionales de las moléculas [15-16]. La utilidad de esta técnica se debe principalmente a su sensibilidad para dar información sobre la identidad química o molecular [17-19], estados de carga [7,12], procesos en interfaces, orden estructural [7, 12-20]. Es por esto que la espectroscopia Raman proyecta un gran mercado para aplicaciones de análisis en medicina/ nanomedicina [18], tecnología de alimentos [20], industria química y petroquímica [20], industria de materiales $[16,21]$, biocombustibles $[7,9,22$, 23]. Pese al gran potencial, actualmente hay una necesidad de concebir sistemas de análisis Raman de fácil uso, económicos y portátiles [17].

Al iluminar una sustancia sólida, líquida o gaseosa, pueden darse de manera independiente o simulatánea dos situaciones, la primera, que parte de la luz inicial sea absorbida por la sustancia y que otra parte sea reflejada. El segundo caso es cuando en la luz reflejada aparecen además colores o cambios de tonalidad luego de haber interactuado con la sustancia. Estos dos marcos globales de interacción han dado lugar a un gran número de aplicaciones de la luz como base para técnicas de análisis de tipo espectroscópico $[14,17]$. En especial, en la espectroscopia Raman se ilumina la muestra preferiblemente con un solo color (luz monocromática) o luz láser [18-20]. Como efecto de la interacción de la luz láser con la materia se observa que la luz reflejada es del mismo color y que además aparecen colores de intensidad muchísimo menor que la original. Las nuevas tonalidades traen información única o "huella digital molecular" sobre aspectos moleculares de la sustancia iluminada [15, 18-22]. Es aquí donde herramientas basadas en el análisis de la luz reflejada se denominan espectroscopias ópticas. La ventaja de una herramienta óptica es por su versatilidad ya que en general no es necesario preparar la muestra. Entre las técnicas espectroscópicas ópticas empleadas comúnmente para analizar biocombustibles se encuentran la absorción UV-vis y de infrarrojo [2427]. Particularmente, una técnica óptica no invasiva que ha venido ganando una posición importante en el análisis de sustancias líquidas o sólidas es la espectroscopia Raman [16-23, 28-30]. La que puede suministrar información química detallada sobre muestras de biocombustibles, y con esto la cantidad porcentual de la mezcla con diésel. Entre las ventajas de la espectroscopia Raman es que la medida se obtiene directamente y sin preparación previa de la muestra [17-20, 22-23,30]. Además, el espectro Raman revela una gran cantidad de características i.e. huella digital molecular, bandas espectrales más detalladas, mayor resolución energética e identidad química o alteración del biocombustible [7, 22-23,30]. En especial en este trabajo se reporta un procedimiento novedoso para obtener espectros Raman de señal magnificada (SERS: Surface Enhanced Raman Spectroscopy) de muestras de biodiésel en diésel.

\section{ANÁLISIS TEÓRICO}

\section{Generalidades de la espectroscopia Raman y montaje experimetal}

La espectroscopia Raman es un método de caracterización óptico no invasivo ni destructivo, el que se basa en la interacción inelástica entre la luz o fotones y la materia, dando como resultado información directa sobre las propiedades vibracionales de las moléculas de la muestra en análisis. El espectro vibracional de cualquier muestra i.e. gaseosa, líquida o sólida es único, y cualquier alteración molecular se ve reflejado en este [18-20,31].

Los fundamentos principales de la espectroscopia Raman pueden ser explicados de manera clásica o con teoría cuántica [15-16]. En el segundo caso la descripción es más concisa debido a que describe aspectos limitados por la teoría clásica i.e. Rotación molecular, relación entre la polarizabilidad inducida y las propiedades moleculares, entre otras. 
El fenómeno de interacción radiación materia que da lugar a la espectroscopia Raman se resume en la Figura 2. El proceso parte con: I) una muestra sólida, líquida o gaseosa en un estado energético inicial p.e. el estado base.

II) La muestra se estimula con luz, para el caso de la espectroscopia Raman se utilza un láser, y cuya energía se da por medio de $\mathrm{E}=\hbar \omega_{\mathrm{exc}}$, donde $\hbar$ es la constante de Planck dividida entre $2 \pi$ y $\omega_{\text {exc }}$ la frecuencia angular del láser.

La luz láser tiene la ventaja de tener una energía muy bien definida, de tal modo que luego del proceso de interacción se puede suprimir con un filtro, quedando solamente aquella luz cuya energía difiere a la de excitación. La luz filtrada es la que guarda información sobre las propiedades moleculares [16].

Cuando la energía de la excitación es igual o supera la brecha óptica energética del material, ocurre una transición electrónica desde el estado base hasta el estado electrónico excitado. En la interacción la muestra experimenta varias situaciones: 1) absorbe parte de los fotones incidentes, 2) emite luz con igual energía a la de excitación $\left(\hbar \omega_{\text {exc }}\right)$, también denominada dispersión de Rayleigh, 3) los estados vibracionales previos a la excitación pueden experimentar transiciones alrededor del estado electrónico inicial, lo que resulta en dispersión de luz de diferente energía a la de excitación.

Analizando más en detalle el proceso III, se distingue que la muestra al retornar a su estado inicial tendría estados vibracionales modificados energéticamente. Si el estado vibracional pasa a un estado de mayor energía, significa que la muestra ganó una fracción de energía en la interacción. Haciendo consideraciones de conservación de la energía, el excedente energético equivale a la energía de la vibración, y el fotón emitido tendría menor energía la que se da por $\hbar\left(\omega_{\mathrm{exc}}-\omega_{1}\right)$, este proceso se denomina dispersión de Stokes [15-16]. En el caso que el estado vibracional quede con menor energía a la de su estado inicial, el fotón dispersado tendría
I)

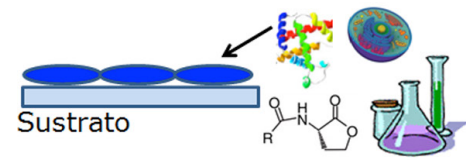

Muestra líquida, sólida, gaseosa orgánica, inorgánica o biológica

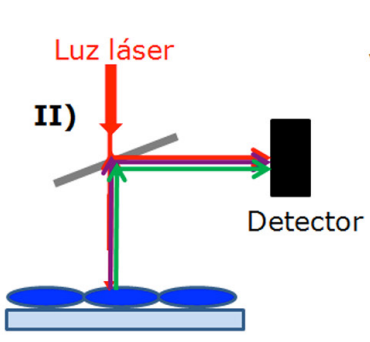

Estados molecualres:

Vibracionales, electrónicos

III)

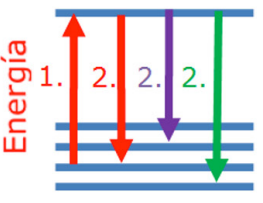

1. Transición óptica

2. Dispersión elástica (Rayleigh)

2. Dispersión inelástica (Raman): Stokes

2. Dispersión inelástica (Raman): anti-Stokes

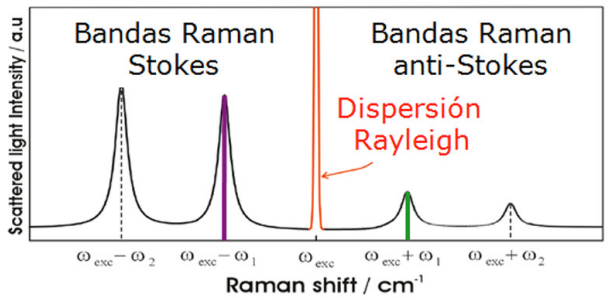

Figura 2. Esquema del proceso de interacción Raman. I) Muestras gaseosa, líquida o sólida. II) Muestra estimulada con luz láser para inducir una transición óptica, la que decae provocando dispersión de la luz láser con energía igual (Rayleigh), menor (Stokes) o mayor (anti-Stokes) que la energía inicial del láser. III) Esquema del espectro Raman con las bandas de Stokes, anti-Stokes y dispersión Rayleigh. 
una energía mayor, la que está dada por $\hbar\left(\omega_{\mathrm{exc}}+\right.$ $\left.\omega_{1}\right)$, este proceso se denomina dispersión de antiStokes [15-16].

Probabilísticamente las dispersiones de Stokes y de anti-Stokes tienen la posibilidad de ocurrencia, solo que la primera es más probable [15-16].

Experimentalmente se observa que la dispersión Stokes es de mayor intensidad que la anti-Stokes, pero ambas señales son mucho más débiles frente a la dispersión Rayleigh [16]. El espectro obtenido con las dispersiones de Stokes y de anti-Stokes dan lugar al denominado espectro Raman, el que se indica en la Figura 2-III.

\section{Montaje experimetal}

En la Figura 3 (a) se muestra un esquema del procedimiento realizado para medir los espectros Raman en biocombustibles. La luz dispersada es recolectada en un espectrofotómetro, el cual consta de filtros knotch que bloquean la luz láser dispersada, seguidamente la señal se enfoca por medio de espejos en una rendija holográfica de difracción de alta densidad, y finalmente llega a una cámara CCD (charged coupled device). La señal obtenida en la CCD pasa al computador, donde se obtiene el espectro de la muestra en análisis. El portamuestras donde se coloca el biodiésel se indica esquemáticamente en la Figura 3 (b). El portamuestra o chip suministrado por la empresa QUBITeXp consta de una capa metálica de Au o Ag de aproximadamente $100 \mathrm{~nm}$ de espesor depositada por evaporación en condiciones de alto vacío sobre sustratos de vidro o de silicio. Posteriomente la capa se nanoestructura por fotolitografía. Los metales Au y Ag poseen propiedades plasmónicas [21, 28-29, 31] apropiadas para el acople energético con el láser. Adicionalmente al ser una superficie metálica rugosa, permite que la señal Raman se magnifique y resulte en el denominado efecto SERS (SERS: Surface Enhaced Raman Spectroscopy) [21, 28-29, 31]. La ventaja de utilizar una superficie metálica nanoestructurada para el análisis del biodésel se debe

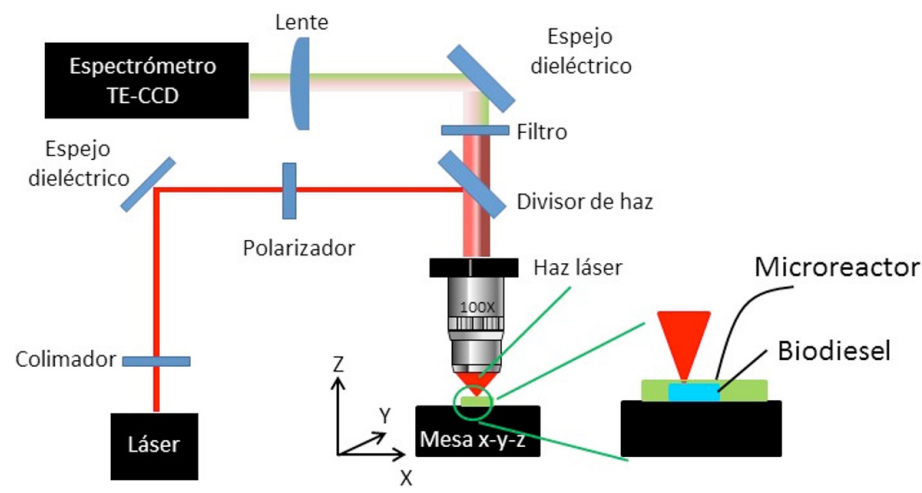

(a)

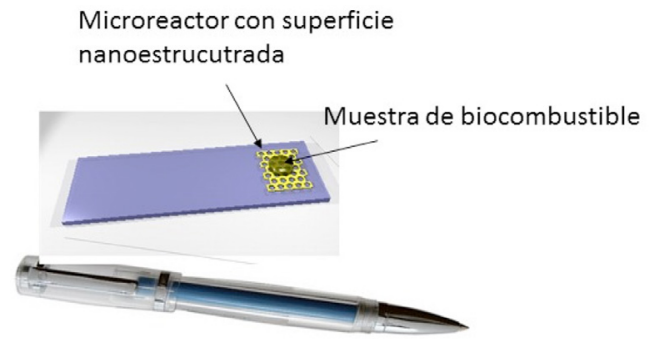

(b)

Figura 3. Esquema experimental. (a) Diagrama del espectrómetro Raman y (b) Esquema de chip para posicionamuento de la muestra de biodésel del análisis nanofotónico por espectroscopia Raman. 
a que la señal Raman puede incrementarse en unas 1000 veces [21,28-29,31]. Esto además hace que el tiempo de medición se reduzca a unos pocos minutos.

Las muestras de biocombustible B100 y diésel fueron suministradas por el Prof. Andrés F. Suárez de la Universidad de Bogotá Jorge Tadeo Lozano, posteriormente se realizaron distintas mezclas en la plancha de agitación durante una hora a temperatura constante. Las mediciones Raman se efectuaron en un equipo fabricado por la empresa QUBITeXp International Trade S.A.S [17]. El espectrofotómetro Raman consta de un una mesa XYZ donde se ubica la muestra la que es excitada con un láser de $658 \mathrm{~nm}$ enfocada con varios objetivos o por fibra óptica.

La luz retrodispersada proveniente de la muestra es filtrada con un filtro tipo notch y posteriormente enfocada en un espectrómetro de alta resolución. La luz Raman entra al espectrómetro por una rejilla de 50 $\mu \mathrm{m}$, posteriormente la luz dispersada se hace incidir sobre una rejilla holográfica de reflexión de 1.800 líneas $/ \mathrm{mm}$. La luz reflejada por la rejilla se enfoca en una cámara CCD que se encuentra refrigerada por un elemento termoeléctrico. Finalmente la señal es integrada y acumulada para ser enviada al computador por puerto USB.

\section{RESULTADOS}

Señales de fotoluminiscencia, fluorescencia, fosforescencia y Raman

La fotoluminiscencia, fluorescencia, fosforescencia y Raman comparten un aspecto en común, la excitación se hace por medio de luz. Generalmente como fuente de estímulo se utiliza una luz láser, la cual posee una energía bien definida con variaciones pequeñas. Cuando se dice que un láser es de $658,0 \mathrm{~nm} \pm 0,5 \mathrm{~nm}$, significa que el color más intenso está en $658,0 \mathrm{~nm}$ y que puede variar su tonalidad entre $657,5 \mathrm{~nm}$ y $658,5 \mathrm{~nm}$. Para el ojo humano estos cambios de tonalidad son imperceptibles e incluso aún con variaciones en $5 \mathrm{~nm}$ de la longitud de onda. En la Figura 4 se muestra parte del espectro electromagnético que corresponde con el visible. Se puede ver claramente que las tonalidades de un tipo de color varían notoriamente.

Para determinar el tipo de moléculas que componen una sustancia, es necesario estimularlas con longitudes de onda bien definidas para saber con qué tipo de color ellas modifican la luz de excitación.

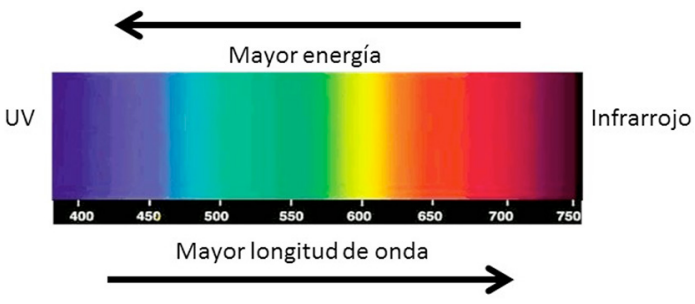

Figura 4. Espectro electromagnético visible. Hay una relación inversa entre la longitud de onda del color y la energía.

Muchos experimentos han mostrado que la luz al pasar o incidir sobre una sustancia, da lugar a una variedad de colores y se han considerado como la huella molecular de la muestra. Esta idea ha sido utilizada en química, medicina, biología, física, ingeniería y muchas otras áreas para determinar de qué está hecha una sustancia.

El biodiésel puede ser obtenido de aceites vegetales o animales, por lo que es interesante conocer si hay rastros del aceite de origen en la muestra final de biodiésel. Con base en la interacción de la radiación con la materia, en especial, en la Figura 5 se indican varios tipos de aceites y biodiésel vistos a la luz del

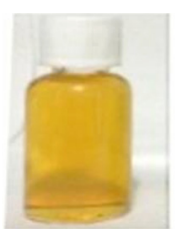

1.

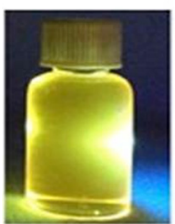

1. Aceite para automóvil

2. Biodiésel

3. Aceite de canola

4. Aceite para lubricación doméstica

Figura 5. Respuesta óptica de aceites y biocombustibles al ser expuestos a (a) luz del espectro visible y (b) luz láser de $405 \mathrm{~nm}$. 
día (iluminación con todo el espectro visible) y su respuesta cuando son iluminados en la oscuridad con luz láser (una sola longitud de onda). En el primer caso algunos aceites se ven muy similares, mientras que en el segundo se observa un cambio de tonalidad en respuesta a la interacción molecular de la sustancia con el láser.

En la Figura 5 (b) se observa claramente que tanto el biodiésel como los aceites responden de una manera muy particular al ser iluminados con el láser. Por tanto, es aquí donde es necesario recurrir a un método de análisis fotónico de la luz dispersada. Por ello, utilizando el mismo montaje descrito en la parte experimental, un láser de excitación de $405 \mathrm{~nm}$, y utilizando una rendija de 100 o de 300 líneas/mm se midió la emisión de cada muestra. En la Figura 6 se presenta el espectro de luminiscencia de cada uno de los aceites y del biocombustible. Cada uno de los espectros de fotoluminiscencia conserva una identidad unívoca con el tipo de muestra. Pero con muchas similitudes en el perfil espectral de las sustancias precursoras del biodiésel. Por tanto, es pertinente recurrir a un refinamiento en la resolución espectral. Esto se logra con la espectroscopia Raman.

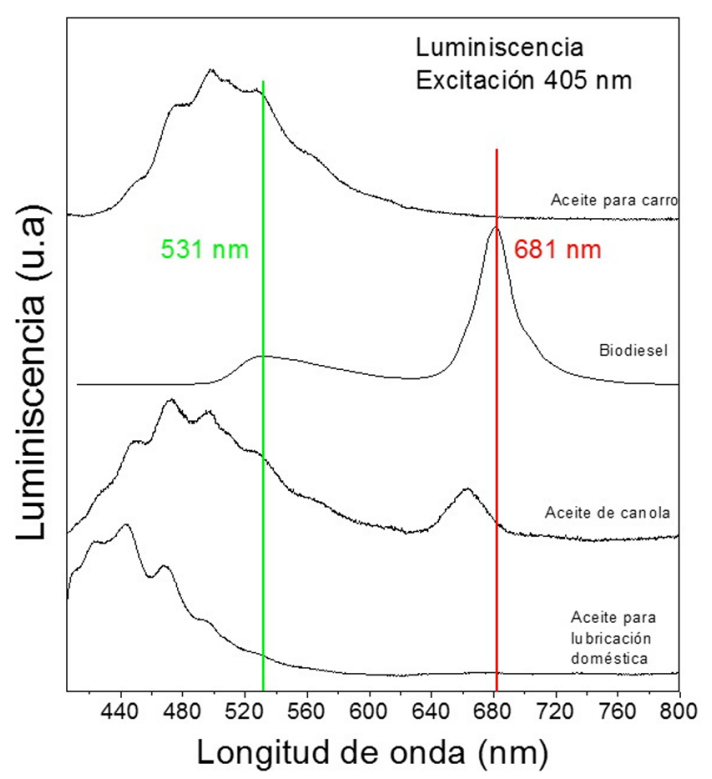

Figura 6. Espectro de fotoluminiscencia de varios aceites y de biodiésel al ser excitados con un láser de estado sólido de $405 \mathrm{~nm}$ a $5 \mathrm{~mW}$.
¿Por qué es tan útil la espectroscopia Raman sabiendo que el cambio de tonalidad de la luz de excitación no se percibe fácilmente? Podría hacerse la siguiente analogía, para subir una escalera se requiere energía (excitación), en el caso de la fotoluminiscencia, fluorescencia y fosforescencia es como si se diera de un solo paso, un salto desde un escalón hasta el más alto (absorción del color o energía de excitación), y simultáneamente hiciéramos dos regresos, uno hasta algún escalón del intermedio (emisión de color con menor energía) y otro al escalón inicial (emisión del mismo color de excitación). La diferencia entre estas tres técnicas se debe principalmente al tiempo (t) de duración que prevalece el cambio de color cuando se suprime la luz de excitación. Experimentalmente se observa que $t_{\text {fotoluminiscencia }}$ $<\mathrm{t}_{\text {fluorescencia }}<\mathrm{t}_{\text {fosforescencia }}$.

En la fosforescencia la emisión de luz luego de apagarse la excitación puede prevalecer varias horas, mientras que en la fluorescencia es del orden de $10^{-6}$ $\mathrm{s}$ y en la fotoluminiscencia del orden de $10^{-9} \mathrm{~s}$. En el efecto Raman los pasos de regreso son prácticamente cercanos y alrededor del escalón inicial, esto da lugar a que las variaciones de la tonalidad del color de excitación sean muy pequeñas, pero con la ventaja que son más precisos al indicar la variación energética entre escalones. Estos cambios sutiles en la señal Raman dan información precisa sobre la estructura molecular, un cambio mínimo en una molécula, se refleja en su espectro Raman. En contraste con las otras técnicas espectroscópicas, estas variaciones pasarían prácticamente desapercibidas.

La espectroscopia Raman al ser altamente sensible, puede detectar cambios muy pequeños i.e. menores que el $1 \%$ en la combinación de sustancias. Por tanto, en las mezclas de biodiésel en diésel, donde un cambio porcentual de la participación de ambos combustibles es notorio en el correspondiente espectro Raman, por esta razón se suele decir que el espectro Raman da la huella molecular de las sustancias.

\section{Análisis basado en nanofotónica para determinar el contenido de biodiésel en diésel}

Las mezclas de biodiésel en diésel fueron colocadas en el microrreactor de paredes nanoestructuradas indicado en la Figura 3 (b). La superficie nanoestructurada del portamuestra permitió obtener espectros Raman magnificados [28-29, 31] como se indica en la Figura 7. 
$\mathrm{Al}$ tener una superficie nanoestructurada hace que el campo eléctrico local del láser se magnifique y a su vez amplifique la variación de la polarizabilidad del Biodiésel, resultando en el efecto SERS [21,2829,31]. Por tanto el método SERS se convierta en una herramienta nanofotónica para el análisis de los biocombustibles. El tipo de sustratos empleados en este análisis dan espectros Raman claros, con una relación señal/ruido favorable para el análisis de Biodiésel en diésel, contrario a las mediciones Raman reportadas por otros autores [30, 22, 23, 30, 32].

La intensidad integrada se determinó por medio de un perfil Lorentuiano de la forma

$$
\mathrm{I}=\mathrm{I}_{0}-\frac{\mathrm{A}}{\mathrm{W}} \sqrt{\pi / 2} \mathrm{e}^{-2\left(\frac{\mathrm{V}-\mathrm{V}_{\mathrm{c}}}{\mathrm{W}}\right)}
$$

con I la intensidad Raman, $\mathrm{I}_{\mathrm{o}}$ una constante que equivale al desplazamiento vertical de la línea base del espectro, A el área de la curva lorentziana y que corresponde con la intensidad integrada, $\mathrm{W}$ ancho del perfil a mitad del pico lorentziano, $X$ corrimiento Raman, y $\mathrm{X}_{\mathrm{c}}$ el centro de la lorentziana.

En la Figura 7(a) se observa que los cambios porcentuales de Biodiésel en diesel afectan el perfil espectral. En especial se analizó la banda a 1.658 $\mathrm{cm}^{-1}$ que corresponde a vibraciones moleculares de compresión tipo cis $\mathrm{C}=\mathrm{C}$ [24, 25, Miran, Abrah].

Esta banda vibracional está asociada con ácidos esteáricos del biodiésel, los que provienen de ácidos grasos saturados presentes en aceites vegetales y grasas animales. Debido a que las muestras analizadas provienen de aceite de palma se identifica ausencia de bandas, las cuales son típicas en biodiésel proveniente de aceites comestibles previamente utilizados en la cocción de alimentos [33].

En la Figura 7 (a) se identifica que para concentraciones bajas de biodiésel en diésel p.e. en B5, la intensidad de la banda es muy baja.

Para lograr una mejor apreciación del efecto porcentual del biodiésel, los espectros de la Figura 7 (a) fueron normalizados y ajustados por medio de funciones lorentzianas para así poder descomponer la banda a $1.658 \mathrm{~cm}^{-1}$ y determinar
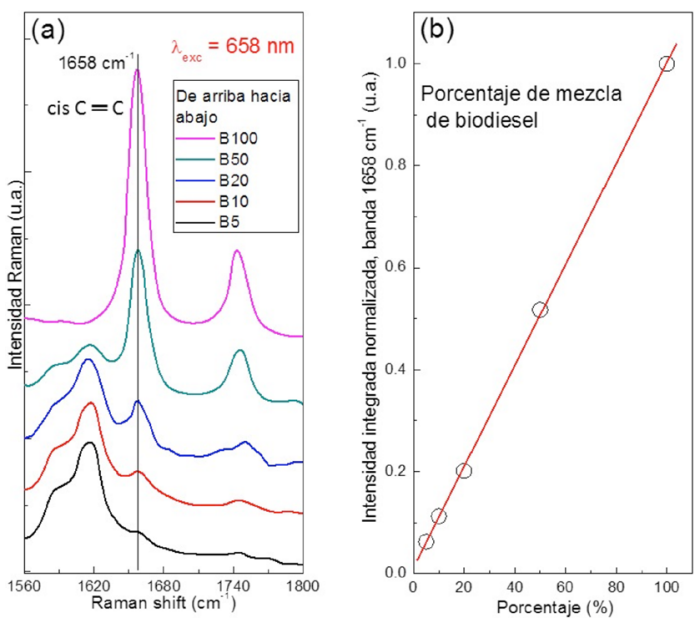

Figura 7. Espectros Raman-SERS de muestras de biodiésel en diésel. (a) De abajo hacia arriba se presentan los espectros de B5, B10, B20, B50, B100, y (b) dependencia de la intensidad integrada de la banda cis $\mathrm{C}=\mathrm{C}$ en dependencia con la concentración de biodiésel en diésel.

su intensidad integrada. En la Figura 7(b) se indica el comportamiento de la intensidad integrada normalizada con la participación porcentual de biodiésel. El diámetro de los círculos corresponde con la incertidumbre de la medida. Se observa que la dependencia es lineal y sirve como vibración molecular de control para determinar el porcentaje de biodiésel.

\section{CONCLUSIONES}

En el presenta artículo se presentó la interacción de la luz láser con el biodiésel como herramienta nanofotónica para determinar la participación porcentual de biodiésel en diésel.

La utilización de aplicaciones basadas en la interacción de la luz con la materia es de gran ayuda en el campo de los biocombustibles. En especial el uso de la técnica de espectroscopia Raman fortalecería el control de calidad de la mezcla de biodiésel en diésel.

Como banda vibracional de control del contenido de biodiésel en diésel se analizó la intensidad de la vibración molecular localizada en $1.658 \mathrm{~cm}^{-1}$, la que corresponde con ácidos esteáricos del biodiésel. 
Se encontró que la intensidad integrada guarda una proporción lineal con el contenido porcentual de biodiésel en diésel.

Para mejorar la baja señal Raman del biodiésel, se implementó un microrreactor con paredes nanoestructuradas, lo que permitió magnificar la señal de los fotones Raman. El efecto se debe a que localmente el campo eléctrico se amplifica y este a su vez aumenta la polarizabilidad del biodiésel. La mayor parte de los fotones Raman provienen de la interacción mediada por la superficie nanoestructurada con las moléculas en contacto directo con la nanoestructura. Por tanto, el análisis Raman de señal magnificada (SERS: SurfaceEnhanced Raman Spectroscopy) se transforma en una herramienta nanofotónica. Un instrumento con estas características permite i) aumentar la señal Raman, ii) mejorar la resolución espectral, iii) reducir el tiempo de diagnóstico, iv) reducir el uso de alta potencia del láser, v) reducir significativamente el ruido en los espectros.

\section{AGRADECIMIENTOS}

A la UMNG en el marco de la línea de investigación de nanofotónica y sensórica.

Los autores expresan su gratitud a la empresa QUBITeXp International Trade S.A.S. por permitir realizar las mediciones ópticas, y al Prof. A.-F. Suárez de la UJTL.

A la Federación Nacional de Biocombustibles de Colombia.

\section{REFERENCIAS}

[1] S. Imtenan, H.H. Masjuki, M. Varman, M.I. Arbab, H. Sajjad, I.M. Rizwanul Fattah, M.J. Abedin, Abu Saeed Md. Hasib. "Emission and performance improvement analysis of Biodiesel-diesel blends with additives". Procedia Engineering. Vol. 90, pp. 472-477. 2014. DOI: 10.1016/j.proeng.2014.11.759.

[2] A.E. Atabani, A.S. Silitonga, Irfan Anjum Badruddin, T.M.I. Mahlia, H.H. Masjuki, S. Mekhilef. "A comprehensive review on biodiesel as an alternative energy resource and its characteristics". Renewable and Sustainable Energy Reviews. Vol. $16 \mathrm{~N}^{\circ} 4$, pp. 2070-2093. 2012. DOI: 10.1016/j. rser.2012.01.003.

[3] D.Y.C. Leung, X. Wu, M.K.H. Leung, "A review on biodiesel production using catalyzed transesterification". Applied Energy. Vol. 87 No 4, pp. 1083-1095. 2010. DOI: $10.1016 /$ j.apenergy.2009.10.006.

[4] P.T. Vasudevan and M. Briggs, "Biodiesel production-current state of the art and challenges", Journal of Industrial Microbiology \& Biotechnology, vol. 35, $\mathrm{N}^{\circ} 5$, pp. 421-430, Jan. 2008. DOI: 10.1007/s10295-008-0312-2.

[5] Distribución del porcentaje de mezcla de Biodiésel en el territorio Colombiano. Julio 2016. [Online] Disponible: http:// www.fedebiocombustibles.com/estadisticamostrar_info-titulo-Biodiesel.htm

[6] J. Xue, T.E. Grift, and A.C. Hansen, "Effect of biodiesel on engine performances and emissions", Renewable and Sustainable Energy Reviews, vol. 15, № 2, pp. 1098-1116, Feb. 2011. DOI: 10.1016/j.rser.2010.11.016.

[7] B.A. Paez-Sierra. "Nanofotónica y sensórica para investigación y desarrollo de biocombustibles". Revista Española de Seguros. Vols. 153-154, pp. 262-284. 2013.

[8] Z. Fan, O. Schröder, and J. Krahl. "Analysis of diesel fuels/biodiesel blends and identification of biodiesel using time-resolved laser-induced fluorescence spectroscopy (TRLFS)". Landbauforschung Applied Agricultural and Forestry Research. Vol. 65, pp. 1-14. 2015.

[9] J. Kiefer. "Recent Advances in the Characterization of Gaseous and Liquid Fuels by Vibrational Spectroscopy". Energies. Vol. 8, pp. 3165-3197. 2015. DOI: 10.3390/ en8043165.

[10] M. Svilans, A. Blūms, R. Kampare. "Spectroscopic monitoring of biodiesel aging". Material Science and Applied Chemistry. Vol. 28, pp. 85-91. 2013. DOI: 10.7250/msac.2013.012.

[11] M. Meira, C.M. Quintella, E.M. de O. Ribeiro, A.K. Guimarães, W. Leal-Silva. "Determination of adulterants in diesel by multivariate calibration associated with LED spectrofluorimetry". International Journal of Chemistry. Vol. $6 \mathrm{~N}^{\circ}$ 4, pp. 24-30. 2014. DOI: $10.5539 / \mathrm{ijc} . \mathrm{v} 6 \mathrm{n} 4 \mathrm{p} 24$.

[12] J.-B. Kim, J.-H. Lee, C.-K. Moon, S.-Y. Kim, J.-J. Kim. "Highly enhanced light extraction 
from surface plasmonic loss minimized organic light-emitting diodes". Advanced Materials. Vol. 25, pp. 3571-3577. 2013. DOI: $10.1002 /$ adma.201205233.

[13] S.V. Gaponenko. "Introduction to Nanophotonics", Cambridge University Press. Cambridge, England, pp. 1.2. 2010. ISBN: 0521763754.

[14] R.P. Prasankumar, A.J. Taylor. "Optical Techniques for Solid-State Materials Characterization". CRC Press. Boca Raton, USA. pp. 3-329. 2011. ISBN: 1439815372.

[15] D.A. Long. "The Raman effect: A unified treatment of the theory of Raman scattering by molecules". John Wiley \& Sons Ltd. Chichester, England, pp. 1-145. 2002. ISBN: 0-471-49028-8.

[16] M.S. Amer. "Raman Spectroscopy, Fullerenes and Nanotechnology". RSC Nanoscience \& Nanotechnology. Cambridge, England. Vol. 13, pp. 43-108. 2010. ISBN: 978-184755-240-2. 20.

[17] Espectrómetros para análisis de Biodiésel. [Online] Disponible: http://www.qubitexp. com, 2016.

[18] P. Matousek, M.D. Morris. "Emerging Raman applications and techniques in biomedical and pharmaceutical fields". Springer. Berlin, Germany, pp. 1-24. 2010. ISBN: 3-642-02648-6.

[19] M.S. Amer. "Raman spectroscopy for soft matter applications". John Wiley \& Sons, Inc., pp. 1-29. New Jersey, USA. ISBN: 978-0-470-45383-4. 2009.

[20] S. Šašic. "Pharmaceutical applications of Raman spectroscopy", John Wiley \& Sons, Inc. New Jersey, USA, pp. 117-156. 2008. ISBN: 0813810132.

[21] B.-A. Paez-Sierra, D.-M. Marulanda, H. Rodríguez. "Interface formation between pentacene and silver contacts investigated by surface-enhanced Raman spectroscopy". Proceedings of SPIE. Vol. 918521, pp. 1-7. 2014. DOI: 10.1117/12.2062367.

[22] O. Samek, A. Jonáš, Z. Pilát, P. Zemánek, L. Nedbal, J. Tríska, P. Kotas and M. Trtílek. "Raman microspectroscopy of individual algal cells: Sensing unsaturation of storage lipids in vivo". Sensors. Vol. 10, pp. 8635-8651. 2010. DOI: 10.3390/ s100908635.
[23] J. Abrahamsson, E. Andreasson, N. Hansson, D. Sandström, E. Wennberg, M. Maréchal, A. Martinelli. "A Raman spectroscopic approach to investigate the production of biodiesel from soybean oil using 1-alkyl3-methylimidazolium ionic liquids with intermediate chain length". Applied Energy. Vol. 154, pp. 763-770. 2015. DOI: 10.1016/j. apenergy.2015.04.123.

[24] M. Insausti, A.A. Gomes, F.V. Cruz, M.F. Pistonesi, M.C.U. Araujo, R.K.H. Galvão, C.F. Pereira, B.S.F. Band. "Screening analysis of biodiesel feedstock using UVvis, NIR and synchronous fluorescence spectrometries and the successive projections algorithm". Talanta. Vol. 97, pp. 579-583. 2012. DOI: $10.1016 / \mathrm{j}$. talanta.2012.04.056.

[25] R.M. Balabin, R.Z. Safieva. "Biodiesel classification by base stock type (vegetable oil) using near infrared spectroscopy data". Anal Chim. Acta Vol. 689, pp. 190-197. 2011. DOI: 10.1016/j.aca.2011.01.041.

[26] G. Veras, A.A. Gomes, A.C. Silva, A.L.B. Brito, P.B.A. Almeida, E.P. Medeiros. "Classification of biodiesel using NIR spectrometry and multivariate techniques". Talanta. Vol. 83, pp. 565-568. 2010. DOI: 10.1016/j.talanta.2010.09.060.

[27] G. Veras, A.L.B. Brito, A.C. Silva, P. Silva, G.B. Costa, L.C.N. Félix. "Biodiesel classification in the visible region". Quim. Nova. Vol. 35, pp. 315-318. 2012. DOI: 10.1590/S0100-40422012000200015.

[28] R. Aroca, "Surface enhanced vibrational spectroscopy", John Wiley \& Sons, Inc. New Jersey, USA, pp. 35-101. 2006. ISBN: 978-0-471-60731-1.

[29] K. Kneipp, "Surface-Enhanced Raman Scattering: Physics and applications". Springer-Verlag. Heidelberg, Germany. Topics in Applied Physics Vol. 103, pp. 1-85. 2006. ISBN: 10: 3540335668.

[30] A.M. Miranda, E.W. Castilho-Almeida, E.H. Martins Ferreira, G.F. Moreira, C.A. Achete, R.A.S.Z. Armond, H.F. Dos Santos, A. Jorio. "Line shape analysis of the Raman spectra from pure and mixed biofuels esters compounds". Fuel. Vol. 115, pp. 118-125. 2013. DOI: $10.1016 / \mathrm{j}$. fuel.2013.06.038. 
[31] Ch. Wölf, "Organic Electronics: Structural and electronic properties of OFETs", Wiley-VCH Verlag GmbH \& Co. Weinheim, Germany, pp. 263-279. 2009. ISBN: 352740810X.

[32] Z. Fang, R.L. Smith, X. Qi, "Production of Biofuels and Chemicals with Microwave",
Springer. Heidelberg, Germany, pp. 145-168. 2015. ISBN: 9401796114.

[33] L. Qu, Z. Wang, and J. Zhang, "Influence of waste cooking oil biodiesel on oxidation reactivity and nanostructure of particulate matter from diesel engine". Fuel. Vol. 181, pp. 389-395. Oct. 2016. 